\title{
Recent Trends and Future Directions in Research Regarding Parents with Intellectual and Developmental Disabilities
}

\author{
Maurice A. Feldman ${ }^{1}$ (D) Marjorie Aunos ${ }^{1}$ \\ Published online: 30 June 2020 \\ (C) Springer Nature Switzerland AG 2020
}

\begin{abstract}
Purpose of Review This report updates research on parents with IDD and their children published since 2014. Recent Findings Since 2014, a plethora of studies using large administrative databases in different countries support a contextual approach to understand why parents with IDD and their children may have worse outcomes than other families. In most studies, increased risk of ill health in women with IDD and health and developmental problems in their children were fully or partially accounted for by socioeconomic and psychosocial hardships. New research has found that pregnant women with IDD tend to have risk factors for pregnancy, birth, and postpartum complications that may contribute to adverse child outcomes. Intervention research is gradually becoming more contextualized.

Summary More studies are needed on multicultural aspects of parenting, programs that could help parents with IDD overcome social and health disadvantages, comprehensive and coordinated service models that start during pregnancy, innovative parent support arrangements, parenting education for teens and young adults with IDD, use of technology, and dissemination and implementation of evidence-based programs.
\end{abstract}

Keywords Parents with intellectual and developmental disabilities $\cdot$ At-risk children $\cdot$ Administrative datasets $\cdot$ Contextual model . Vulnerable parent groups

\section{Introduction}

Research on parenting by persons with intellectual and developmental disabilities (IDD) ${ }^{1}$ has gone through several stages as described by Llewellyn and Hindmarsh [1]. This report examines trends in this field in the 5 years since their report. Early research was concerned about effects on the children [2, 3 ], the over-representation of children of parents with IDD in child protection [4], and teaching parenting skills to parents with IDD [5]. Then, through quantitative and qualitative

\footnotetext{
${ }^{1}$ This term will be used to include persons with study labels of intellectual disability, intellectual or cognitive impairment, learning difficulties, learning disabilities (UK), or borderline intellectual levels. Many of the studies in this area use different definitions and measures to determine the intellectual level of their participants and rarely document formal ID diagnosis.
}

This article is part of the Topical Collection on Intellectual Disability

Maurice A. Feldman

mfeldman@brocku.ca

1 Department of Applied Disability Studies, Brock University, 1812 Sir Isaac Brock Way, St. Catharines, ON L2S 3A1, Canada analyses, the focus shifted to identifying socioeconomic and psychosocial determinants of parenting and child problems, including child protection involvement [6]. A more nuanced contextual view, such as the one proposed by Feldman [7•], has come to dominate the research focus. The contextual model has been expanded to include antenatal, birth, and postpartum factors that could affect initial parenting abilities and early child outcomes [8]. Intervention research has moved beyond parent education and is beginning to incorporate a contextual approach and addressing gaps in previous studies.

\section{Extending Support for Contextual Models}

Feldman's [7•] contextual-interactional model (see Fig. 1) adapted well-established ecological viewpoints of family life by Bronfenbrenner [9], Sameroff [10], Belsky [11], and others for families headed by parents with IDD. For many years, researchers and advocates were dismayed by the dominance of a univariate model of parenting by persons with IDD [12]. This simplistic view argues that parents with IDD are inherently incapable of adequate parenting and would not benefit 


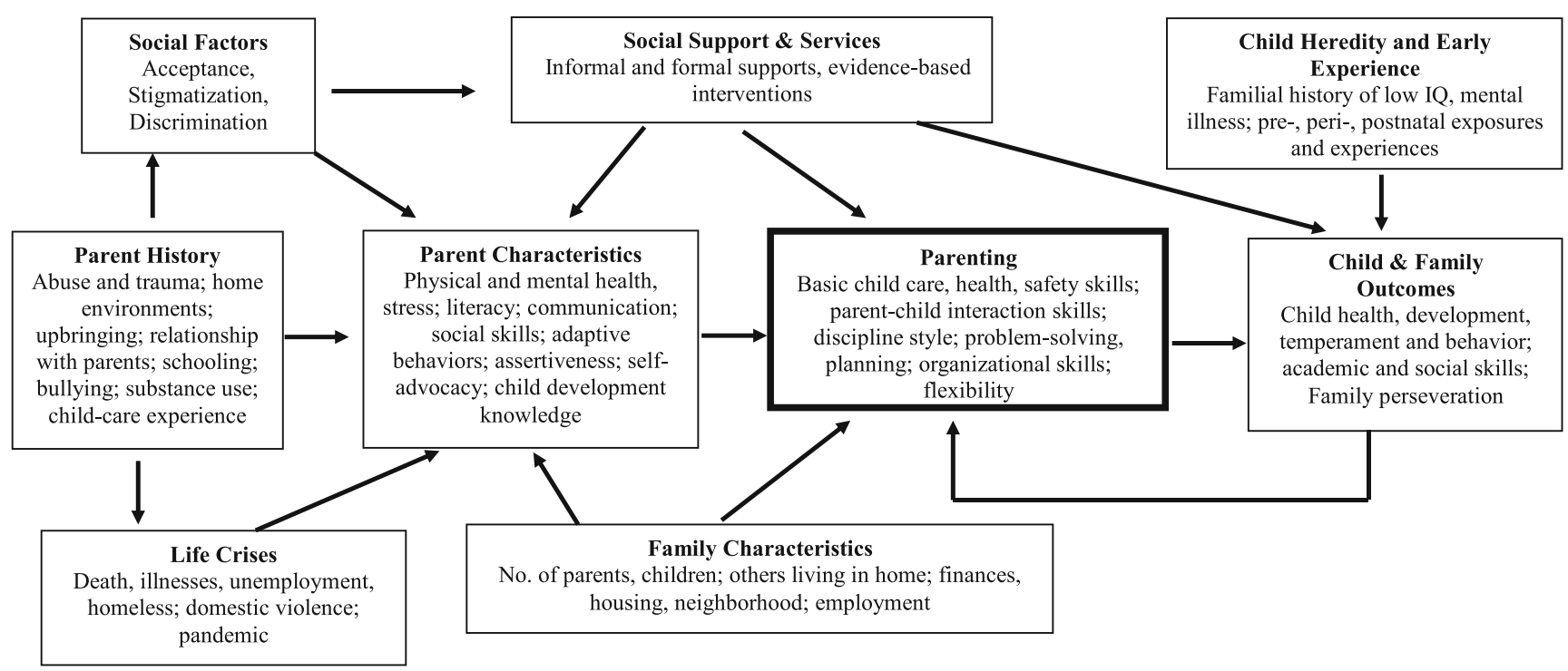

Fig. 1 Interaction model of parenting for parents with learning difficulties (adapted from Feldman, 2002; reprinted with permission of NADD)

from parent education because of their low intelligence. The overshadowing of parental cognitive disability over other factors that may better predict parenting abilities, such as parent history of trauma $[13 \bullet, 14]$, parental mental health, and social support $[15,16]$, has contributed to discriminatory child protection decision-making and legal outcomes regarding child custody when parents have IDD $[17,18]$. As can be seen in Fig. 1, numerous variables potentially are involved and interrelated in determining parenting and child and family outcomes. Several specific variables are listed in each panel that can impede or support successful parenting, and parent, family, and child outcomes. For instance, societal factors such as stigmatization and discrimination of persons with disabilities (top left panel of the model), coupled with a parent's history of successfully disguising their disability, may result in the parent eschewing needed services and supports to avoid admitting they need help. This action could reduce likelihood of parenting success, thereby adversely impacting child health and development. Child protection workers may identify the parent as uncooperative and blaming the parent's lack of insight as to their support needs on their cognitive disability rather than a strong motivation to hide their disability. Another example of not looking beyond the parent's disability would be to blame inadequate newborn care and apathy to the child on the mother's IDD, rather than considering the possibility that she may have postpartum depression. At the time the model was first proposed in 2002 [7•], many of these relationships were hypothetical with respect to families with parents who have IDD. Since then, considerable evidence has emerged supporting the model. The remainder of this section summarizes recent research relevant to the model since the Llewellyn and Hindmarsh [1] report that provided evidence in support of a contextual approach to parenting by parents with IDD.
Evidence from large datasets has expanded support for a contextual model of parenting for these families. Emerson et al. [19•] used the UK Understanding Society longitudinal database of over 14,000 families to compare socioeconomic disadvantage and the health status of parents with and without IDD (several hundred parents had IDD with the exact sample size depending on age cohort). Parents with IDD had poorer health and socioeconomic hardship but controlling for socioeconomic factors eliminated the health differences between parents with and without IDD.

Powell and associates ran a series of secondary analyses of the US Fragile Families and Child Wellbeing Study subsamples of 1298 mothers without IDD and 263 mothers with IDD of 3-year old children. Like in the UK [19•], US mothers with IDD had significantly more health and socioeconomic hardships. As both poor parental health and low socioeconomic level are associated with inadequate parenting and child developmental problems [21, 22], the authors surmised that these factors should be considered when trying to understand parenting issues in parents with IDD.

Several studies that included child outcome data provide further support for a contextual model [16, 23•, 24, 25••]. Wade et al. [16] showed that in a sample of 120 Australian parents with IDD, social support and parental mental health played key roles in parenting practices and child developmental outcomes, consistent with results reported in a randomized Canadian child protection sample of 1170 parents with IDD [15]. Wade et al. [16] found that social support mediated the relationship between measures of socioeconomic disadvantage and parent mental health. The relationship between parental mental health and child outcomes was mediated by (self-reported) parent's warmth and child-care practices. Meppelder, Hodes, Kef, and Schuengel [23•] studied the interplay of parental stress, social support, and child behavior 
problems in 134 Dutch parents with IDD and their 1 to 7 -yearold children. They found that large social support networks mediated the relationship between child-related parental stress and (teacher or worker) ratings of child behavior problems. Another study using the US Fragile Families and Child Wellbeing dataset reported no significant differences in having asthma or being overweight/obese in 3-year-old children of mothers with and without IDD [24]. While children of mothers with IDD were rated as being less healthy, controlling for family socioeconomic hardship, maternal health, stress, and social support eliminated the relationship of maternal IDD and child health problem scores. Hindmarsh, Llewellyn, and Emerson [25••] conducted secondary analyses of the UK Millennium Cohort Study when children were 3 $(N=15,590), 5(N=15,246)$ and 7 years old $(N=13,857)$. Sample sizes of participating mothers with IDD were 64, 57, and 46 in the 3-, 5- and 7-year-old child age groups, respectively. Child wellbeing scores were significantly lower in the parental IDD group at 3 and 5 but not 7 years old. Adjusting for contextual variables such as child illness, poverty and maternal mental health significantly reduced risk to the children of parents with IDD and eliminated significant differences with children who had parents without IDD.

Wickström, Höglund, Larsson, and Lundgren [26] used Sweden birth registry information from 1999 to 2012 that included 478,577 children, 2749 of whom had birth mothers with IDD. Like Hindmarsh et al. [25••], Wickström et al. [26] found risk reduction to children when controlling for parental factors such as mental health and substance use. However, Wickström et al. [26] still reported significant risk of injuries and child abuse to children of Swedish mothers with IDD even after adjusting for these maternal characteristics and some child factors (e.g., gestational age, epilepsy). It is interesting that these researchers did not control for external contextual variables included in other studies such as social support and economic disadvantage that found larger decreases in risk to children of parents with IDD [1,16,24,25•]. While caution must be exercised comparing results across studies from different countries using different databases and measures, the disparate findings of the Wickström et al. [26] study suggest, among other things, that social support and poverty may contribute important variance to child outcomes and/or there is a dose effect - the more disadvantages, the more risk [27].

Although the above studies show the influence of contextual factors on explaining differential outcomes of children with and without parents with IDD, there is still much unaccounted variance. Researchers have begun to look at earlier potential determinants of adverse child outcomes. Several independent research teams using birth cohort data in the US, Canada, UK, and Sweden have reported remarkably similar findings - mainly that compared to women without IDD, women with IDD smoke more during pregnancy, are younger, experience problems during pregnancy and delivery, and are less apt to access or benefit from prenatal care. Their newborns are at significant risk for adverse birth outcomes such as preterm, low birth weight, stillborn, perinatal death, and

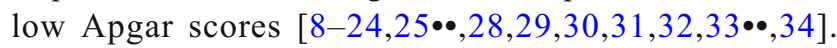
Furthermore, women with IDD are more likely to have postpartum physical, neurological, and mental health problems than new mothers without IDD that could affect the care of their newborns $[35,36 \bullet, 37]$. These recent large-scale studies are beginning to identify reasons why young children of mothers with IDD may show early developmental delay [3,15-24,25••,38]. This research highlights the need for services that focus on prenatal and postnatal health and wellbeing of women with IDD [32, 39]. Conceptual models should include prenatal maternal health, delivery, and postnatal complications as additional determinants of child outcomes.

\section{Recent Trends in Interventions for Parents with IDD and Their Children}

This section will briefly review some of the current directions of intervention research for these families. As evidence mounts supporting a contextual view of families headed by parents with IDD, are assessments and interventions adopting a more contextualized stance? Feldman and Aunos [40] have manualized a comprehensive, competence-based parenting capacity assessment approach based on Feldman's [7•] contextual model coupled with direct observation of parenting skills and an empirical test of responsiveness to evidencebased parent training. In a case in Massachusetts in which a custody decision to remove a child of a mother with IDD was based primarily on her perceived intellectual inability to raise children, in 2015, the US Department of Justice and US Department of Health and Human Services [41 ••] jointly issued guidelines which specified that contextually based parenting capacity assessments, emulating Feldman and Aunos' model [40], for parents with IDD was necessary to meet the legal requirements of the Americans with Disabilities Act. Unlike typical parenting capacity assessments, recommendations from comprehensive and contextual assessments usually do not come to a definitive conclusion that the parent can or cannot parent. A contextualized assessment identifies impediments and supports for the parent to be successful and may make service and support suggestions beyond parent education [40]. For instance, recognizing the importance of social support, a contextual assessor may recommend teaching the parent to build a support network, be more socially engaged, and participate in activities that promote parent, child, and family wellbeing.

Unfortunately, few intervention studies have focused on building social support and wellbeing in parents with IDD. In one of the first studies attempting to decrease social isolation of mothers with IDD, McGaw, Ball, and Clark [42] 
conducted a control study of a 14-week social group for 12 British parents with IDD. They found that compared to the control group $(n=10)$, the training group increased their social networks, friendships, and self-confidence. However, these improvements did not impact the parents' self-reported relationships with their children and there were no direct observations of the impact of the intervention on parent-child interactions. In Canada, McConnell et al. [43] employed an uncontrolled, pre-post, mix-methods design to evaluate the Social Learning Program (SLP) that is intended to increase community engagement and psychological health of parents with IDD. The SLP included individual goal setting, problemsolving, and feedback as well as group activities. Different from other intervention studies involving parents with IDD, McConnell et al. [43] primarily recruited from agencies supporting adults with fetal alcohol spectrum disorder (FASD) and included aboriginal parents. Of the 33 parents originally recruited, 18 mothers with IDD completed the full study. Pre-post scores on the Depression, Anxiety and Stress Scale [39] showed significant improvements in depression, anxiety, and stress, with moderate effect sizes. No pre-post differences were found on self-report measures of social support and personal control. Several themes emerged from qualitative analysis, including awareness there were other parents like them, making and helping new friends, being more socially engaged, enjoying social interactions, recognizing strengths, accomplishing goals, being more confident and having a more positive outlook. Gustavsson and Starke [40] qualitatively reported that a parenting group in Sweden reduced the parents' social isolation, built friendships, and more trusting relationships with their social workers. The results of these preliminary group intervention studies to increase social supports and wellbeing in parents with IDD are encouraging, but more controlled studies are needed that include measures that demonstrate the impact of these approaches on parenting success, parent-child interactions, and child and family outcomes.

While there is now considerable evidence that behaviorally based parent training programs, using audio-visual materials, modeling, coaching, and positive reinforcement, can improve parenting skills in parents with IDD with corresponding benefits to the children [5, 46, 47], little research has been conducted on collateral effects of the intervention on the parents. Three recent parent training studies that focused on reducing child problem behavior and increasing positive parent-child interactions showed pre-post training decreases in parenting stress related to the child $[48 \bullet, 49,50]$. Tahir et al. [50] showed increased parenting self-efficacy and decreased reported use of punishment in two mothers with IDD who received individualized game-based training.

Another trend in the intervention literature is to adapt existing parent training programs for parents with IDD. Recent studies have shown promising but variable results, for adaptations of Triple $\mathrm{P}$ - positive parenting program [51], parent-child interaction therapy [52], and positive behavior support [49]. An RCT of an adaptation of video-feedback intervention to promote positive parenting and sensitive discipline did not significantly improve ratings of targeted parenting skills [53]. Modifications to the original programs included making the material more concrete; less reliance on reading, writing, and detailed explanations; slower pace and extended sessions; and more frequent positive feedback. These features are already part of programs designed specifically for parents with IDD [54]. Variable findings of these program adaptations may be due to trying to adhere to original program components that do not work as well with parents with IDD (e.g., group sessions) or indirect measures of parent and child behaviors (e.g., self-report questionnaires, rating scales) rather than direct counts of parent and child behaviors).

An emerging trend is to start imparting parenting knowledge to teens and young adults with IDD before they become parents. Pearson, Chaisty, and Stenfert Kroese [55] recruited 25 students from special needs schools (mean age $=18$ years, mean IQ $=59$ ) to watch an 8-min informational DVD about parent-child attachment and ask questions. A seven-item questionnaire testing knowledge gleaned from the video was given pre-post viewing (same day). The participants then were given an information booklet to take home. Two to 3 weeks later, they were retested. There were significant increases in correct responses on the posttest and follow-up test from the pretest. The change scores were not significantly correlated with participant IQ score or other demographic characteristics. There was no test to see if the participants would interact with a baby any differently after training. A feasibility study in Sweden recruited six special needs high school students to take an 8week parenting course using a parenting toolkit developed in the Netherlands by Marje Hodes [56]. The students also had to care for a programmable baby simulator for 3 days and nights. Taking care of the "baby" proved difficult for the participants, with only three of them being able to care for the baby for the 3 -day trial, and then only at 54\% correct care according to the data downloaded from the simulators. Scores on parenting attitude and self-efficacy questionnaires did not change significantly. Although the preliminary results were weak, the researchers concluded that it was feasible to mount a full-scale study using the simulator and toolkit. While it makes sense to give older teens and young adults with IDD some exposure to parenting, it remains to be seen how these programs affect teenage pregnancy and initial parenting knowledge and competence.

\section{Future Directions}

This section identifies some future research needs in expanding the contextual-interactional model and assessment 
and interventions based on recent findings in support of the model reviewed above.

Intergenerational Research The contextual-interactional model as presented in Fig. 1 posits some intergenerational effects that are now supported by recent research-for example, the impact of parental childhood trauma on parenting, child, and family outcomes $[13 \bullet, 14-56,57 \bullet, 58]$. More research is needed to investigate mechanisms of intergenerational transmission and how children and grandchildren are impacted by positive and negative life events of the parent in question [59].

Assessment Research The research on assessment practices involving parents with IDD suggests the system is fraught with bias and invalid methods [17, 18]. The comprehensive, contextual parenting assessment approach [40] based on the contextual-interactional model is now recommended as best practice [41••], but more research is needed to evaluate the validity and impact of these assessments. For instance, to what extent does implementation of the contextual assessment recommendations lead to better outcomes for families than the traditional assessment model that focuses on parental cognitive abilities as a proxy for parenting skills? Research is needed on developing a simple screening tool that can help workers quickly identify which parents may need accommodations such as pictorial rather than textual materials.

Multinational Collaboration and Diversification Support for a contextual approach has benefited from secondary analyses of large administrative databases. However, these databases were not specifically designed to focus on families with parents with IDD and used different definitions, recruitment methods, measures and analyses. These differences make interpretation of results across studies problematic. It would be worthwhile to design a purpose-built multinational study with consistent objectives and research protocols. Perhaps, the Special Interest Research Group of the International Association for the Scientific Study of Intellectual and Developmental Disabilities could take the lead to plan such a study.

International comparative studies, in addition to national inquiries into minority cultures [60], could study the impact of distinct cultural practices. For instance, some cultures promote or even force marriage and pregnancy in young women while other cultures actively discourage or even prevent procreation by persons with disabilities. As such, the context of the pregnancy (e.g., choice, imposed, accidental, discouraged) might impact how parenting is perceived and supported. To inform policies and services, future research could examine the application of the interactional model [7•] to a broader population of parents that are viewed (usually wrongly) as being at risk for poor parenting. Such parents include those with other types of disabilities besides IDD (e.g., physical, sensory, mental illness) as well as inexperienced (e.g., teenage) parents.

Parent and Child Perspectives The refinement of the contextual model would benefit from continuing inclusion of the parents' and children's voices. For instance, asking parents how they negotiate and choose their support network members could help organize supports that are more responsive to the parents' needs and preferences. While numerous qualitative studies have either interviewed parents (usually mothers) with IDD or offspring [61, 62], more studies are needed that interview mothers, fathers, and children from the same families to gain a better understanding of their views of their parent-child relationships and the impact of parental disability on family life.

Despite multiple adversities faced by parents with IDD, many of them are still parenting and living fruitful lives. The fields of positive psychology and resilience [63] may contribute to research on parenting and parents with IDD in determining how personal attributes and specific historical and current experiences build self-esteem, confidence, positive parent-child relationships, and more effective parenting practices. Research in this field should become more participatory, involving collaboration with the parents with IDD and their children not only as the subjects of studies, but also as research collaborators themselves [64, 65].

Interventions More than 40 years of research has demonstrated the effectiveness of behaviorally oriented parent training in increasing parenting skills in parents with IDD [5, 46, 47, 54]. New well-designed studies could examine the use of technology (e.g., reminder texts, smartphone apps, video modeling and feedback, telehealth parent training, virtual reality). For instance, a parent could practice positive behavior support strategies to deal with child misbehavior using a virtual reality device and receive feedback from a parent trainer before trying the strategies with the child. Studies examining telehealth parent training [66] for parents with IDD are needed to reach parents living in remote areas, who prefer that nobody comes to their homes, or are in lockdown due to a pandemic. Parent training strategies, like general case training [67], should be tested that promote generalization and long-term impact of parent training programs on child and parent wellbeing and family preservation. More studies could focus on the consequences of contextually based comprehensive interventions to reduce economic and psychosocial hardships seen in these families. Research could explore how training might support women with IDD in establishing and maintaining healthy relationships and social support networks to reduce their risk of domestic violence and exploitation.

The notions of peer support [68] and co-parenting arrangements [69] need further exploration. In terms of parents with IDD, peer support involves other parents providing parent 
training in addition to instrumental and emotional support. Co-parenting involves another person or couple (related or unrelated to the mother and father with IDD) who share parenting responsibilities with the biological parents. Understanding the circumstances under which these relationships may work and what factors are needed for success might offer child protection authorities alternatives to permanent removal of the child while allowing the parents with IDD to raise their child. More research is needed on expanding the initial studies [55] of innovative approaches to educate teens and young adults with IDD about healthy and safe sex and relationships, family planning, pregnancy, and parent education before they become parents. This education may reduce current risk of adverse pre-, peri-, and postnatal mother and baby outcomes described above $[24,28,30]$.

Implementation While a more thorough understanding of parenting determinants and research to continue to build evidence-based intervention programs are necessary, further study is needed on the dissemination and implementation of this knowledge. We need to better understand how to make adapted and effective interdisciplinary services available and accessible in communities to those parents who need them. For instance, how can service pathways be developed and coordinated to include accessible family planning, perinatal care, maternal health, housing, and parent education in order to decrease the health disparities in mothers and their newborns $[8,29-32,33 \bullet]$ ? Parenting assessment procedures and intervention programs need to fit within current service systems, as well as be offered by skillful professionals. More studies are needed on training of social workers and clinical psychologists on assessing and intervening with parents with IDD. This topic could be embedded into social work curricula and CPS in-service training [70] so that workers become more comfortable interacting with these parents and are aware of evidence-based practices. Likewise, psychologists conducting parenting capacity assessments should be obliged to take workshops on adapting assessments for parents who have IDD, using a comprehensive, contextual approach [40] rather than the current emphasis on cognitive disability evaluations as a proxy for direct measures of parenting competence [18].

Evaluations of current child protection practices, the effects of contextually based parenting assessments (when used), and the decision-process of judges [40] may help to eliminate biases in the current system that result in premature and possibly unwarranted child removal and failure to provide necessary supports to keep the child with their biological parents. Certainly, to help guide court decisions towards family preservation, the availability of evidencebased services and supports would be essential, as judges might be more inclined to keep families together knowing they could receive ongoing supports that adjust to changing needs of the family.

\section{Conclusion}

Research in parenting by persons with IDD has grown in sophistication and design. We now have considerable knowledge of contextual variables affecting parent, child, and family outcomes. Blaming perceived and real parenting problems and removing the child based only on the parent's IDD should be considered ethically objectionable. Yet, both authors as well as our colleagues in numerous countries frequently come across child protection cases that maintain this practice. Specific service pathways geared to supporting families with multiple impediments to parenting starting before conception might offer parents and children the opportunity to grow together in safe and nurturing families, as promoted by the United Nations Conventions on the Rights of Children, and Persons with Disabilities [71, 72].

\section{Compliance with Ethical Standards}

Conflict of Interest The authors declare that they have no conflicts of interest.

Human and Animal Rights and Informed Consent This article does not contain any studies with human or animal subjects performed by any of the authors.

\section{References}

Papers of particular interest, published recently, have been highlighted as:

- Of importance

• Of major importance

1. Llewellyn G, Hindmarsh G. Parents with intellectual disability in a population context. Curr Dev Disord Rep. 2015;2:119-26. https:// doi.org/10.1007/s40474-015-0042-x.

2. Reed EW, Reed SC. Mental retardation: a family study. Philadelphia: Saunders; 1965.

3. Feldman MA, Case L, Towns F, Betel J. Parent education project I: the development and nurturance of children of mentally handicapped mothers. Am J Ment Def. 1985;90:253-8.

4. Hayman RL. Presumptions of justice: law, politics and the mentally retarded parent. Harvard Law Rev. 1990;103:1201-74. https://doi. org/10.2307/1341412.

5. Feldman MA. Parenting education for parents with intellectual disabilities: a review of outcome studies. Res Dev Disabil. 1994;15: 299-332. https://doi.org/10.1016/0891-4222(94)90009-4.

6. McConnell D, Aunos M, Feldman M, Prasad NG. Parental cognitive impairment and child maltreatment in Canada. Child Abuse Negl. 2011;35:621-32. https://doi.org/10.1016/j.chiabu.2011.04. 005 .

7. Feldman MA. Parents with intellectual disabilities and their children: impediments and supports. In: Griffiths D, Federoff P, editors. Ethical dilemmas: sexuality and developmental disability. Kingston, New York: NADD Press; 2002. p. 255-292. This book chapter describes the contextual model of parenting for parents with IDD that spurred much current research. 
8. Brown HK, Cobigo V, Lunsky Y, Vigod SN. Maternal and offspring outcomes in women with intellectual and developmental disabilities: a population-based cohort study. Brit J Obstet Gynaecol. 2017;124:757-65. https://doi.org/10.1111/1471-0528. 14120.

9. Bronfenbrenner U, Ceci SJ. Nature-nurture reconceptualised in developmental perspective: a bioecological model. Psychol Rev. 1994;101:568-86. https://doi.org/10.1037/0033-295x.101.4.568.

10. Sameroff AJ, Chandler M. Reproductive risk and the continuum of caretaking causality. In: F. Horowitz, editor. Review of child development research (Vol. 4). Chicago, IL: University of Chicago Press. 1975. p. 157-243.

11. Belsky J. Etiology of child maltreatment: a developmentalecological analysis. Psychol Bull. 1993;114:413-34. https://doi. org/10.1037/0033-2909.114.3.413.

12. Booth T, Booth W. Parents with learning difficulties in the child protection system: experiences and perspectives. J Intellect Disabil. 2005;9:109-29. https://doi.org/10.1177/1744629505053922.

13. Lindberg L, Fransson M, Forslund T, Springer L, Granqvist P. Maternal sensitivity in mothers with mild intellectual disabilities is related to experiences of maltreatment and predictive of child attachment: a matched-comparison study. J Appl Res Intellect Disabil. 2017;30:445-55. https://doi.org/10.1111/jar.12300 This study and [14] identify parental history of trauma as a key predictor of parenting problems.

14. McGaw S, Scully T, Pritchard C. Predicting the unpredictable? Identifying high-risk versus low-risk parents with intellectual disabilities. Child Abuse Negl. 2010;34:699-710. https://doi.org/10. 1016/j.chiabu.2010.02.006.

15. Feldman MA, McConnell D, Aunos M. Parental cognitive impairment, mental health and child outcomes in a child protection population. J Ment Health Res Intellect Disabil. 2012;5:66-90. https:// doi.org/10.1080/19315864.2011.587632.

16. Wade C, Llewellyn G, Matthews J. Parent mental health as a mediator of contextual effects on parents with intellectual disabilities and their children. Clin Psychologist. 2015;19:28-38. https://doi. org/10.1111/cp.12055.

17. Booth T, Booth W, McConnell D. The prevalence and outcomes of care proceedings involving parents with learning difficulties in the family courts. J Appl Res Intellect Disabil. 2005;18:7-17. https:// doi.org/10.1111/j.1468-3148.2004.00204.x.

18. Callow E, Tahir M, Feldman MA. Judicial reliance on parental IQ in appellate level child welfare cases involving parents with intellectual and developmental disabilities. J Appl Res Intellect Disabil. 2017;30:553-62. https://doi.org/10.1111/jar.12296.

19. Emerson E, Llewellyn G, Hatton C, Hindmarsh G, Robertson J, Man WYN, et al. The health of parents with and without intellectual impairment in the UK. J Intellect Disabil Res. 2015;59:1142-54. https://doi.org/10.1111/jir.12218 While parents with IDD were shown to have poorer health than parents without IDD, controlling for socioeconomic hardships eliminated the between group differences in health outcomes.

20. Powell RM, Parish SL, Akobirshoev I. The health and economic well-being of US mothers with intellectual impairments. J Appl Res Intellect Disabil. 2017;30:456-68. https://doi.org/10.1111/jar. 12308 .

21. Lefmann T, Combs-Orme T. Prenatal stress, poverty, and child outcomes. Child Adol Soc Work J. 2014;31:577-90. https://doi. org/10.1007/s10560-014-0340-x.

22. Shelleby EC. Economic stress in fragile families: pathways to parent and child maladjustment. J Child Fam Stud. 2018;27:3877-86. https://doi.org/10.1007/s10826-018-1232-z.

23. Meppelder M, Hodes M, Kef S, Schuengel C. Parenting stress and child behaviour problems among parents with intellectual disabilities: the buffering role of resources. J Intellect Disabil Res. 2015;59:664-77. https://doi.org/10.1111/jir.12170 This study shows the important role of social support in mediating the relationship between parent stress and child problems.

24. Powell RM, Parish SL, Akobirshoev I. Health of young children whose mothers have intellectual disability. Am J Intellect Dev Disabil. 2016;121:281-94. https://doi.org/10.1352/1944-7558121.4.281.

25.• Hindmarsh G, Llewellyn G, Emerson E. The social-emotional wellbeing of children of mothers with intellectual impairment: a population-based analysis. J Appl Res Intellect Disabil. 2017;30: 469-81. https://doi.org/10.1111/jar.12306 Analyzing a large longitudinal data set, this study showed that problems in children who had parents with IDD were mitigated when controlling for socioeconomic disadvantage (see also [24]).

26. Wickström M, Höglund B, Larsson M, Lundgren M. Increased risk for mental illness, injuries, and violence in children born to mothers with intellectual disability: a register study in Sweden during 1999 2012. Child Abuse Negl. 2017;65:124-31. https://doi.org/10.1016/ j.chiabu.2017.01.003.

27. McConnell D, Feldman M, Aunos M, Pacheco L. Child welfare process and outcomes for children of parents with cognitive impairment [II]: findings from the CIS_2008. Family and Disability Studies, University of Alberta. http://www.fdsa.ualberta.ca, 2017.

28. Akobirshoev I, Parish SL, Mitra M, Rosenthal E. Birth outcomes among US women with intellectual and developmental disabilities. Disabil Health J. 2017;10:406-12. https://doi.org/10.1016/j.dhjo. 2017.02.010.

29. Brown HK, Kirkham YA, Cobigo V, Lunsky Y, Vigod SN. Labour and delivery interventions in women with intellectual and developmental disabilities: a population-based cohort study. J Epidem Comm Health. 2016;70:238-44. https://doi.org/10.1136/jech2015-206426.

30. Höglund B, Larsson M. Pregnancy, birth outcomes and perinatal health of children of women with ID. J Intellect Disabil Res. 2019;63:780-0.

31. Malouf R, Henderson J, Redshaw M. Access and quality of maternity care for disabled women during pregnancy, birth and the postnatal period in England: data from a national survey. BMJ Open. 2017;7:e016757. https://doi.org/10.1136/bmjopen-2017-016757.

32. Mitra M, Akobirshoev I, Parish S. Severe maternal morbidity among women with intellectual and developmental disabilities: a population based study. J Intellect Disabil Res. 2019;63:781-1.

33.• Mitra M, Parish SL, Clements KM, Cui X, Diop H. Pregnancy outcomes among women with intellectual and developmental disabilities. Am J Prev Med. 2015;48:300-8. https://doi.org/10.1016/j. amepre.2014.09.032 The first study to show that US mothers with IDD and their newborns were at increased risk of adverse outcomes including premature deliveries, low birth weight and low Apgar scores.

34. Parish SL, Mitra M, Son E, Bonardi A, Swoboda PT, Igdalsky L. Pregnancy outcomes among US women with intellectual and developmental disabilities. Am J Intellect Dev Disabil. 2015;120: 433-43. https://doi.org/10.1352/1944-7558-120.5.433.

35. Mitra M. Postpartum health of women with intellectual and developmental disabilities: a call to action. J Women's Health. 2017;26: 1-2. https://doi.org/10.1089/jwh.2017.6382.

36. Brown HK, Cobigo V, Lunsky Y, Vigod SN. Postpartum acute care utilization among women with intellectual and developmental disabilities. J Women Health. 2017;26:329-37. https://doi.org/10. 1089/jwh.2016.5979 This study showed that new mothers with IDD were more likely to have postpartum depression and other mental health problems that may not be considered when judging their parenting abilities and interactions with their babies.

37. Brown HK, Lunsky Y, Wilton AS, Cobigo V, Vigod SN. Pregnancy in women with intellectual and developmental disabilities. J Obst Gynaecol Can. 2016;38:9-16. https://doi.org/10.1016/ j.jogc.2015.10.004. 
38. Emerson E, Brigham P. The developmental health of children of parents with intellectual disabilities: cross sectional study. Res Dev Disabil. 2014;35:917-21. https://doi.org/10.1016/j.ridd.2014.01. 006.

39. Homeyard C, Montgomery E, Chinn D, Patelarou E. Current evidence on antenatal care provision for women with intellectual disabilities: a systematic review. Midwifery. 2016;32:45-67. https:// doi.org/10.1016/j.midw.2015.10.002.

40. Feldman MA, Aunos M. Comprehensive competence-based parenting capacity assessment for parents with learning difficulties. Kingston, NY: NADD Press; 2010.

41.• US Department of Justice, US Department of Health and Human Services. Protecting the rights of parents and prospective parents with disabilities: technical assistance for state and local child welfare agencies and courts under Title II of the Americans with Disabilities Act and Section 504 of the Rehabilitation Act. Civil Rights Sector and Administration for Children and Families Office of Civil Rights, Washington, D.C. 2015. https://www.ada. gov/doj_hhs_ta/child_welfare_ta.html. A landmark ruling that has major repercussions for improving parenting capacity assessments of parents with IDD, child custody decisions and the quality of interventions provided in the US.

42. McGaw S, Ball K, Clark A. The effect of group intervention on the relationships of parents with intellectual disabilities. J Appl Res Intellect Disabil. 2002;15:354-66. https://doi.org/10.1046/j.14683148.2002.00143.x.

43. McConnell D, Feldman M, Aunos M, Pacheco L, Savage A, Hahn $\mathrm{L}$, et al. Ameliorating psychosocial risk among mothers with intellectual impairment. Comm Ment Health J. 2016;52:944-53. https:// doi.org/10.1007/s10597-015-9979-9.

44. Lovibond SH, Lovibond P F Manual for the depression anxiety stress scales (2nd ed.). Sydney: The Psychology Foundation of Australia Inc. 1995.

45. Gustavsson M, Starke M. Groups for parents with intellectual disabilities: a qualitative analysis of experiences. J Appl Res Intellect Disabil. 2017;30:638-47. https://doi.org/10.1111/jar.12258.

46. Wade C, Llewellyn G, Matthews J. Review of parent training interventions for parents with intellectual disability. J Appl Res Intellect Disabil. 2008;21:351-66. https://doi.org/10.1111/j.14683148.2008.00449.x.

47. Wilson S, McKenzie K, Quayle E, Murray G. A systematic review of interventions to promote social support and parenting skills in parents with an intellectual disability. Child Care Health Dev. 2013;40:7-19. https://doi.org/10.1111/cch.12023.

48. Hodes MW, Meppelder M, de Moor M, Kef S, Schuengel C. Alleviating parenting stress in parents with intellectual disabilities: a randomized controlled trial of a video-feedback intervention to promote positive parenting. J Appl Res Intellect Disabil. 2017;30: 423-32. https://doi.org/10.1111/jar.12302 A well-controlled study showing that parent training using video feedback reduced childrelated parenting stress in parents with IDD.

49. Knowles C, Blakely A, Hansen S, Machalicek W. Parents with intellectual disabilities experiencing challenging child routines: a pilot study using embedded self-determination practices. J Appl Res Intellect Disabil. 2017;30:433-44. https://doi.org/10.1111/jar. 12303.

50. Tahir M, Sword FMA. Evaluation of a game-based parent education intervention to increase positive parent-child interactions in parents with learning difficulties. Beh Analy: Res Pract. 2015;15: 187-200. https://doi.org/10.1037/bar0000022.

51. Glazemakers I, Deboutte D. Modifying the 'positive parenting program' for parents with intellectual disabilities. J Intellect Disabil Res. 2013;57:616-26. https://doi.org/10.1111/j.1365-2788.2012. 01566.x.

52. Chengappa K, McNeil CB, Norman M, Quetsch LB, Travers RM. Efficacy of parent-child interaction therapy with parents with intellectual disability. Child Fam Behav Ther. 2017;39:253-82. https://doi.org/10.1080/07317107.2017.1375680.

53. Hodes MW, Meppelder M, de Moor M, Kef S, Schuengel C. Effects of video-feedback intervention on harmonious parentchild interaction and sensitive discipline of parents with intellectual disabilities: a randomized controlled trial. Child Care Health Dev. 2018;44:304-11. https://doi.org/10.1111/cch.12506.

54. Feldman MA, Tahir M. Skills training for parents with intellectual disabilities. In: Singh N, editor. Handbook of evidence-based practices for individuals with intellectual disabilities. New York: Springer; 2016. p. 613-31.

55. Pearson T, Chaisty F, Stenfert Kroese B. Teaching young adults with intellectual disabilities about early parent-child attachment behaviors using an educational DVD. Inter J Develop Disabil. 2019 (online). Doi: https://doi.org/10.1080/20473869.2019.1573573

56. Janeslätt G, Larsson M, Wickström M, Springer L, Höglund B. An intervention using the parenting toolkit "children-what does it involve?" and the real-care-baby simulator among students with intellectual disability - a feasibility study. Appl Res Intellect Disabil. 2019;32:380-9. https://doi.org/10.1111/jar.12535.

57. RLB F, AP DP. Intergenerational transmission of trauma: maternal trauma-related cognitions and toddler symptoms. Child Maltreat. 2018;23:126-36. https://doi.org/10.1177/1077559517737376.

58. Granqvist P, Forslund T, Fransson L, Springer L, Lindberg L. Mothers with intellectual disability, their experiences of maltreatment, and their children's attachment representations: a smallgroup matched comparison study. Attach Human Develop. 2014;16:417-36. https://doi.org/10.1080/14616734.2014.926946.

59. Traustadóttir R, Sigurjónsdóttir H. The 'mother' behind the mother: three generations of mothers with intellectual disabilities and their family support networks. Appl Res Intellect Disabil. 2008;21:33140. https://doi.org/10.1111/j.1468-3148.2008.00450.x.

60. Pacheco L, McConnell D. Love and resistance of mothers with intellectual disability from ethnocultural communities in Canada. Appl Res Intellect Disabil. 2017;30:501-10. https://doi.org/10. 1111/jar.12342.

61. Booth T, Booth W. Parents with learning difficulties in the child protection system: experiences and perspectives. J Intellect Disabil. 2005;9:109-29.

62. Collings S, Grace R, Llewellyn G. The role of formal support in the lives of children of mothers with intellectual disability. Appl Res Intellect Disabil. 2017;30:492-500. https://doi.org/10.1111/jar. 12361.

63. Niemiec R, Shogren K, Wehmeyer M. Character strengths and intellectual and developmental disability: a strengths-based approach from positive psychology. Educ Train Autism Develop Disabil. 2017;52:13-25.

64. Frankena T, Naaldenberg J, Cardol M, Linehan C, van Schrojenstein H, de Valk L. Active involvement of people with intellectual disabilities in health research - a structured literature review. Res Develop Disabil. 2015;45-46:271-83. https://doi.org/ 10.1016/j.ridd.2015.08.004.

65. Snelgrove S. Bad, mad and sad: developing a methodology of inclusion and a pedagogy for researching students with intellectual disabilities. Int J Inclusive Educ. 2005;9:313-29.

66. Ferguson J, Craig E, Dounavi K. Telehealth as a model for providing behavior analytic interventions to individuals with autism spectrum disorder: a systematic review. Autism Develop Disorders. 2019;49:582-616. https://doi.org/10.1007/ s10803-018-3724-5.

67. Ward-Horner J, Sturmey P. The effects of general-case training and behavioral skills training on the generalization of parents' use of discrete-trial teaching, child correct responses, and child maladaptive behavior. Behav Intervent. 2008;23:271-84. https://doi.org/10. 1002/bin.268. 
68. Strnadová I, Collings S, Loblinzk J, Danker J. Parents with intellectual disabilities and their perspective of peer support: "it depends on how they give it". Appl Res Intellect Disabil. 2019;32:879-89. https://doi.org/10.1111/jar.12579.

69. McHale J, Sirotkin Y. Coparenting in diverse family systems. In: Bornstein MH, editor. Handbook of parenting, volume 3: being and becoming a parent. 3rd ed. New York: Routledge; 2019. p. 137-66. https://doi.org/10.4324/9780429433214.

70. Laliberte T. Are we prepared? Child welfare work with parents with intellectual and/or developmental disabilities. J Public Child
Welfare. 2013;7:633-57. https://doi.org/10.1080/15548732.2013. 861382.

71. United Nations. Convention on the Rights of the Child. https:// www.ohchr.org/en/professionalinterest/pages/crc.aspx, 1989.

72. United Nations. Convention on the Rights of Persons with Disabilities and Optional Protocol. https://www.un.org/ disabilities/documents/convention/convoptprot-e.pdf, 2008.

Publisher's Note Springer Nature remains neutral with regard to jurisdictional claims in published maps and institutional affiliations. 